\title{
The Tumor Suppressive Role of MiRNA- 509-5p by Targeting FOXM1 in Non-Small Cell Lung Cancer
}

\author{
Ning Ma Weihua Zhang $^{\mathrm{a}}$ Chenhui Qiao ${ }^{\mathrm{a}}$ Hong Luo $^{\mathrm{a}}$ Xin Zhang ${ }^{\mathrm{a}}$ Donghai Liu ${ }^{\mathrm{a}}$ \\ Suhua Zang ${ }^{\text {a }}$ Liang Zhang $^{\text {a Jingyun Baib }}$ \\ aDepartment of Cardiovascular Surgery, the First Affiliated Hospital of Zhengzhou University, \\ Zhengzhou, 'Department of Nephrology, the First Affiliated Hospital of Zhengzhou University, \\ Zhengzhou, China
}

\section{Key Words}

MiRNA-509-5p • FOXM1 • Non-small cell lung cancer • Apoptosis • Migration • Invasion

\begin{abstract}
Background/Aims: Deregulation of microRNAs (miRNAs) expression is a frequent event in cancer development and progression. Recent studies have implied that abnormal expression of miRNAs is frequently observed in non-small cell lung cancer (NSCLC). Here, we examined the levels and biological functions of miR-509-5p in NSCLC. Methods: The levels of miR-509$5 p$ were measured by real-time quantitative PCR (RT-PCR) in NSCLC cell lines and NSCLC tissues along with adjacent normal tissues. Cell viability was analyzed by MTT and colony formation assay. Cell migration and invasion were evaluated by transwell and wound healing assay. In addition, we predicted the putative targets of miR-509-5p by bioinformatics analyses. Moreover, by luciferase-reporter assay, we analyzed the relationship between miR-509-5 $p$ and the target in NSCLC cells. Results: miR-509-5p expression was significantly reduced in NSCLC tissues compared with adjacent normal tissues. In addition, miR-509-5p decreased cell proliferation, migration and invasive capability of NSCLC cells. Moreover, we found that FOXM1 was a putative target of miR-509-5p. Enforced miR-509-5p expression in NSCLC cells reduced both mRNA and protein levels of FOXM1. Furthermore, dual-luciferase reporter assay showed miR-509-5p could bind to the $3^{\prime}$ untranslational regions of FOXM1 mRNA. Furthermore, overexpression of FOXM1 reversed cell viability, migration, invasion and vimentin levels suppressed by miR-509-5p mimics in H1299 cells. Conclusions: miR-509-5p exerts tumor-suppressive effects by attenuating FOXM1 in NSCLC. Collectively, these findings provide further evidence that miR-509-5p may be considered as a novel and potential target for the diagnosis, prognosis and treatment of NSCLC.
\end{abstract}




\section{Cellular Physiology Cell Physiol Biochem 2016;38:1435-1446 \begin{tabular}{l|l} 
DOI: 10.1159/000443086 & $\begin{array}{l}\text { O 2016 The Author(s). Published by S. Karger AG, Basel } \\
\text { www.karger.com/cpb }\end{array}$
\end{tabular} \\ Ma et al.: MiRNA-509-5p Inhibits NSCLC by Targeting FOXM1}

\section{Introduction}

Lung cancer is the leading cause of death worldwide [1]. Non-small cell lung cancer (NSCLC) accounts for approximately $85 \%$ of all lung cancers, and its course of disease is very severe, meaning a poor prognosis for patients [2,3]. Despite great advances in chemotherapy and surgical techniques, the prognosis for NSCLC is still dismal, and the overall 5-year survival rate is about 15\% [1]. The distant metastases are responsible for the failure of lung cancer therapy and the poor prognosis of lung cancer [4]. Therefore, it is urgent to further investigate the underlying mechanisms of NSCLC.

MicroRNAs (miRNAs) are small, 18 25 nucleotides, non-coding RNAs that modulate gene expression post-transcriptionally via inhibiting translation or inducing target mRNA degradation [5]. Aberrant expression of miRNAs occurs in many types of cancers, some of which function as tumor suppressor genes or oncogenes [6]. Some studies have implied that miRNAs act as crucial modulators for cancer cell migration, proliferation or epithelialmesenchymal transition (EMT) [7-14]. miR-509-5p has been demonstrated to inhibit EMTrelated genes in melanomas [15]. Previous studies reported miR-509-5p functioned as a tumor suppressor in breast cancer [16]. Zhang et al. have suggested that miR-509-5p played an important role in renal cell carcinoma (RCC) by inhibiting cell proliferation and migration and promoting cell apoptosis [17]. In addition, miR-509-5p expression was significantly lower in RCC patient plasma compared with normal individuals [17]. Ectopic expression of miR-509-5p inhibited cervical cancer and hepatocellular carcinoma cell proliferation and invasion [18]. However, the role of miR-509-5p in the NSCLC cells remained poorly understood.

In the present study, we found that miR-509-5p was significantly downregulated in NSCLC tissues compared with that in adjacent non-tumor tissues. And the expression of miR-509-5p was inversely correlated with the cell proliferation, migration and invasive capability of NSCLC cells. Additionally, we found that FOXM1 was a putative target of miR509-5p by bioinformatics analysis. Moreover, dual-luciferase reporter assay showed miR509-5p could bind to the 3' untranslational regions of FOXM1 mRNA. Lastly, a significantly negative correlation was found between miR-509-5p and FOXM1 in human NSCLC and normal tissues. Altogether, our results suggested that miR-509-5p was a tumor suppressor in NSCLC, via directly targeting FOXM1.

\section{Materials and Methods}

\section{Human samples}

Human NSCLC tissues and their corresponding adjacent normal tissues were collected at the time of surgical resection from 20 patients with NSCLC from 2013 to 2014 at the First Affiliated Hospital of Zhengzhou University (Zhengzhou, China) with written informed consent of patients according to the hospital's ethics committee. Human tissues were collected after surgery and immediately frozen in liquid nitrogen and stored at $-80^{\circ} \mathrm{C}$ refrigerator for further use as previously described [19].

\section{Cell culture}

Human non-small cell lung cancer cells 293T, HLF, 95-D, A549, H1299, PC-9, H358 and H1650 cells purchased from the American Type Culture Collection (ATCC). HLF, 95-D, A549, H1299, PC-9, H358 and H1650 cells were cultured in RPMI 1640 media (Gibco, Carlsbad, CA) supplemented with 10\% fetal bovine serum (FBS) (Gibco, Carlsbad, CA) as previously described [20]. 293T cells were cultured in DMEM media (Gibco, Carlsbad, CA) supplemented with $10 \% \mathrm{FBS}$. All cells were incubated at $37^{\circ} \mathrm{C}$ in a humidified atmosphere containing $5 \% \mathrm{CO}_{2}$.

Transfection

miR-509-5p mimics, miR-509-5p inhibitor and negtative control (NC) were purchased from GenePharma (Shanghai, China). Cells were trypsinised, counted, and seeded into 6-well plates the day prior 


\section{Cellular Physiology Cell Physiol Biochem 2016;38:1435-1446 and Biochemistry Published online: April 04, $2016 \quad \begin{aligned} & \text { DOI: 10.1159/000443086 } 2016 \text { The Author(s). Published by S. Karger AG, Basel } \\ & \text { www.karger.com/cpb }\end{aligned}$ \\ Ma et al.: MiRNA-509-5p Inhibits NSCLC by Targeting FOXM1}

to transfection to ensure 70\% cell confluence on the day of transfection. Transfection of miRNA mimics/ inhibitors into H1299, A549 cells was performed using lipofectamine 2000 (Invitrogen) in accordance with the manufacturer's advised procedure [21]. The miRNA mimics or inhibitors were used at a final concentration of $60 \mathrm{nM}$. At $48 \mathrm{~h}$ after transfection, RT-PCR and Western blotting were performed. H1299 cells were co-transfected with pcDNA3.1-FOXM1 vectors and miRNA mimics using lipofectamine 2000 (Invitrogen) in accordance with the manufacturer's advised procedure [21]. The miRNA mimics and vectors were used at a final concentration of $60 \mathrm{nM}$ and $2 \mu \mathrm{g}$ respectively.

\section{RNA isolation and real time RT-PCR}

Total RNA was isolated from cells using TRIzol reagent (Invitrogen, USA) and synthesized into cDNA by M-MLV reverse transcriptase (Invitrogen, USA) according to the manufacturer's instructions. Real-time quantitative PCR (RT-PCR) was performed on a BioRad CFX96 Real-Time PCR platform using SYBR Green Master Mix Kit (Roche). Primers were used in this study as follows: miR-509-5p forword primer as: TACTGCAGACAGTGGCAAT, reverse primer as: GTGCAGGGTCCGAGGT; U6 forword primer as: CTCGCTTCGGCAGCACA; reverse primer as: AACGCTTCACGAATTTGCGT. FOXM1 forword primer as: ATACGTGGATTGAGGACCACT, reverse primer as: TCCAATGTCAAGTAGCGGTTG; Vimentin forword primer as: TACAGGAAGCTGCTGGAAGG, reverse primer as: ACCAGAGGGAGTGAATCCAG; $\beta$-actin forword primer as: AAACTGGAACGGTGAAGGTG, reverse primer as AGTGGGGTGGCTTTTAGGAT.

\section{Cell viability assay}

Cells were seeded into 96-well plates at a concentration of $5 \times 10^{3}$ cells per well. Following incubation for 24,48 or $72 \mathrm{~h}, 20 \mu \mathrm{L}$ of MTT $(5.0 \mathrm{mg} / \mathrm{mL})$ was added to each well. Following incubation at $37^{\circ} \mathrm{C}$ for $4 \mathrm{~h}$, $200 \mu \mathrm{L}$ of DMSO (Sigma, USA) was added to each well after removing the medium and MTT from the wells. The absorbance was measured using a micro-plate reader at $490 \mathrm{~nm}$ [22].

\section{Colony formation assay}

To assess colony formation, $24 \mathrm{~h}$ after transfection, 500 cells were plated in 6-well plates and grown for 2 weeks; the culture medium was replaced every 4 days. Cells were fixed with methanol and stained with $0.5 \%$ crystal violet for 20 minutes; visible colonies were counted. Triplicate wells were measured for each group [23].

\section{Migration and invasion assay}

Migration and invasion assays were performed using transwell chambers. For migration assay, $5 \times 10^{4}$ cells were seeded into the upper chamber of transwells (BD Bioscience). For invasion assay, $1 \times 10^{5}$ cells were added into the upper chamber precoated with matrigel (BD Bio-science). In both assays, cells were maintained in medium without serum in the upper chamber, and medium containing $10 \%$ FBS was added to the lower chamber as chemoattractant. After $24 \mathrm{~h}$ incubation, cells that did not migrate or invade through the membrane were wiped out. Then the membranes were fixed and stained with $0.5 \%$ crystal violet. Three random fields were counted per chamber using an inverted microscope (Olympus), and each experiment was repeated three times [24].

\section{Wound healing assay}

Cells were grown to confluence on 6-well plates, and the monolayers were wounded with a P10 micropipette tip. The wound-healing pictures were taken at the indicated time points using an inverted microscope (Olympus). The relative area of wound sealing was calculated using NIH ImageJ software, and the data were summarized based on sextuple assays for each experiment.

miRNA target prediction

The analysis of miR-509-5p predicted targets was performed using the algorithms TargetScan (http:// targetscan.org/), PicTar5 (http://pictar.mdc-berlin.de/) and miRDB (http://www.mirdb.org/miRDB/).

Dual-luciferase activity assay

The human 3'-untranslated region (3'-UTR) of FOXM1 gene was amplified by PCR and cloned into the $\mathrm{XbaI}$ site of the pGL3-Control vector (Promega, Madison, WI, USA), downstream of the luciferase gene, to generate the vector pGL3-FOXM1. For luciferase assay, the 293T and A549 cells were cultured in 24-well plates and transfected with 500 ng of either pGL3- FOXM1 or pGL3-control vector and 50 pmol of miR-509- 


\section{Cellular Physiology Cell Physiol Biochem 2016;38:1435-1446 \begin{tabular}{l|l} 
DOI: 10.1159/000443086 & $\begin{array}{l}\text { O 2016 The Author(s). Published by S. Karger AG, Basel } \\
\text { www.karger.com/cpb }\end{array}$
\end{tabular} \\ Ma et al.: MiRNA-509-5p Inhibits NSCLC by Targeting FOXM1}

5 p mimics or negative controls, respectively. Transfection of miRNAs was carried out using Lipofectamine 2000 in accordance with the manufacturer's procedure (Life Technologies). At $24 \mathrm{~h}$ after transfection, firefly luciferase activity was measured using the Dual-Luciferase Reporter Assay (Promega). The above experiment was repeated at least three times.

\section{Western blotting}

Cells were harvested and resuspended in PBS. After centrifugation at 2000 rpm for 5 minutes, cells were lysed on ice in RIPA buffer. Equivalent amounts of proteins (20 to $40 \mu \mathrm{g}$ ) were separated on $8-12 \%$ SDS-PAGE and transferred to polyvinylidene difluoride membranes (Millipore, Bedford, MA, USA) for antibody blotting. Specific primary antibodies used for Western blotting included FOXM1 (Cat. No. 5436S), Vimentin (Cat. No. 5741S), E-cadherin (Cat. No. 3195S) and $\beta$-Actin (Cat. No. 4967S) antibodies (purchased from Cell Signaling Technology, Danvers, MA, USA) [25].

\section{Statistical analysis}

Results were expressed as mean values SE (standard error). Statistical analysis was performed using one-way analysis of variance (ANOVA) followed by posthoc Dunnett's test for multiple comparisons. A level of $P<0.05$ was considered to be significant. Pearson's correlation coefficient was calculated to analyse the correlation. The Statistical Package for Social Sciences (SPSS) software (version 16.0) was used in this study.

\section{Results}

\section{Expression of miR-509-5p in NSCLC cell lines and tissues}

To examine miR-509-5p expression levels, we detected the levels of miR-509-5p in NSCLC and normal human lung fibroblasts (HLF) cell lines by RT-PCR. As shown in Fig. 1A, miR-509-5p was significantly downregulated in all NSCLC cell lines including 95-D, A549, H1299, PC-9, H358 and H1650 cells, compared with normal lung fibroblast cell line HLF. To further confirm the association between miR-509-5p and NSCLC, we detected the levels of miR-509-5p in 20 paired normal NSCLC and adjacent normal tissues. We found NSCLC tissue specimens exhibited lower miR-509-5p levels compared with adjacent normal tissue specimens (Fig. 1B).

miR-509-5p decreases the cell viability and colony formation capability of NSCLC cells

Previous study has shown that miR-509-5p inhibits cell proliferation in renal cell carcinoma [17]. Therefore, we next tested whether miR-509-5p could attenuate the cell

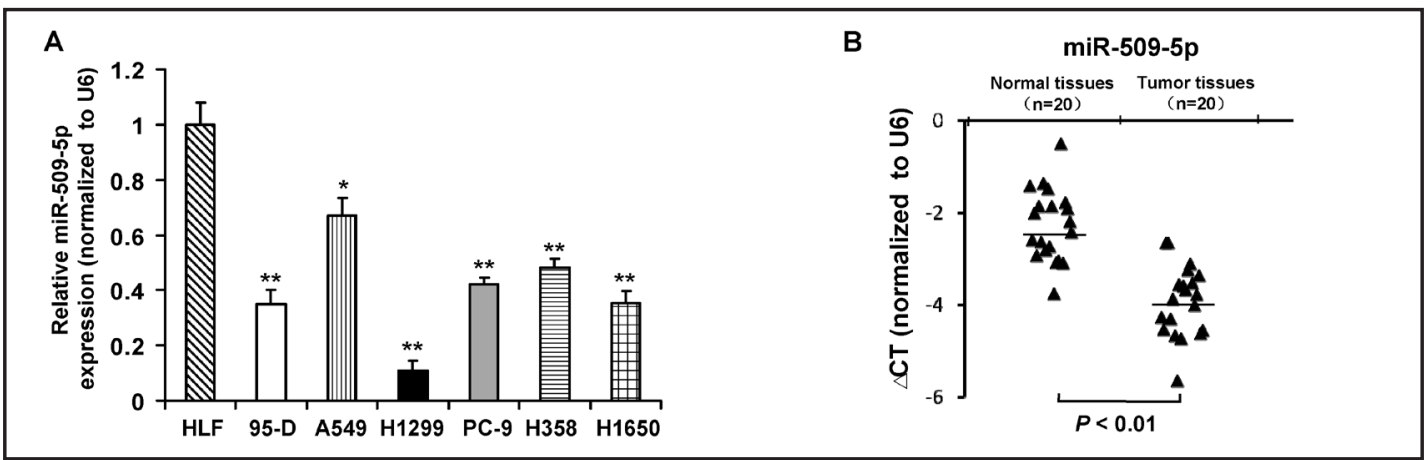

Fig. 1. Expression of miR-509-5p in NSCLC cell lines and tissues. (A) miR-509-5p levels in different NSCLC cell lines were measured by RT-PCR. The experiments were repeated three times. Data represent mean \pm SE. $*, P<0.05,{ }^{* *}, P<0.01$, compared with the HLF cells. (B) miR-509-5p levels were significantly decreased in NSCLC tissues compared with adjacent non-tumor tissues. Expression of mature miR-509-5p was determined by RT-PCR in 20 paired human NSCLC tissues and their corresponding adjacent normal tissues and normalized against an endogenous U6 RNA control. The experiments were repeated three times. 


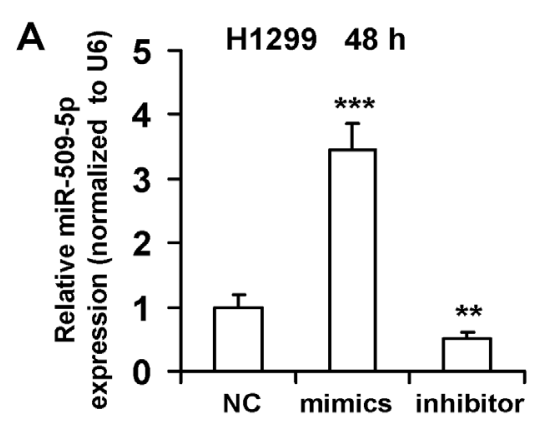

C

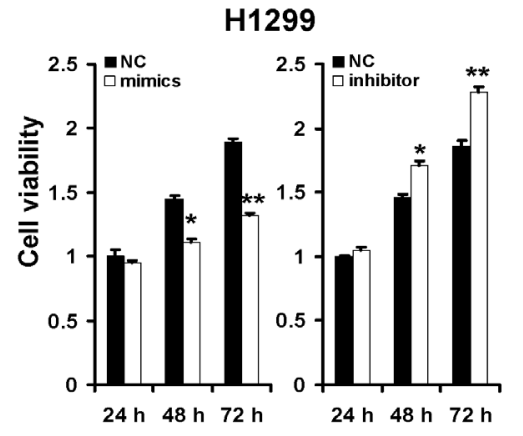

E

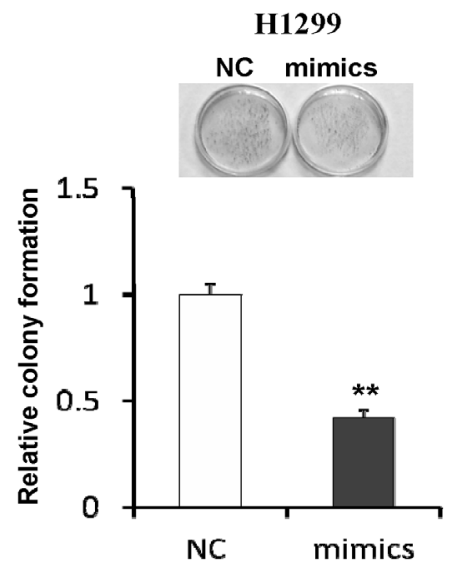

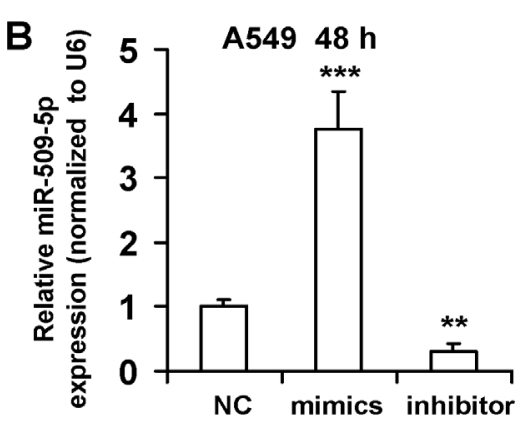

D

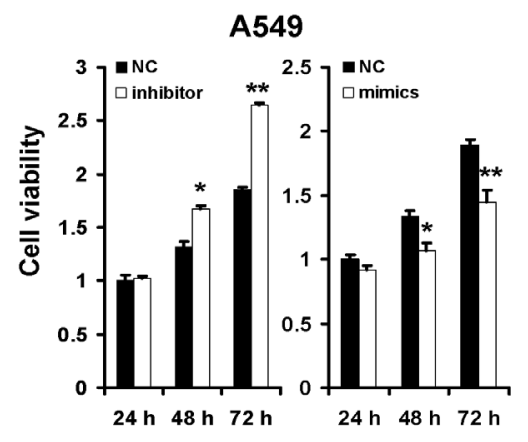

$\mathbf{F}$

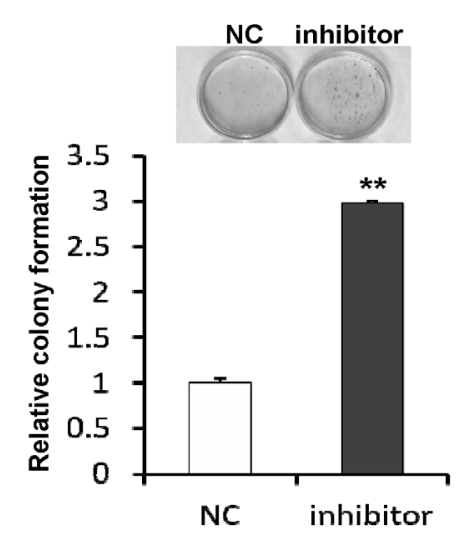

Fig. 2. miR-509-5p decreases the cell viability and colony formation capability of NSCLC cells. (A) and (B) H1299 and A549 cells were transfected with miR-509-5p mimics, miR-509-5p inhibitor and the negative control (NC), respectively. RT-PCR was performed to detect the expression of miR-509-5p. (C) and (D) MTT assay was performed to examine $\mathrm{H} 1299$ and A549 cell viability after transfection at 24, 48 and $72 \mathrm{~h}$, respectively. (E) Transfection of miR-509-5p mimics significantly inhibited H1299 cells colony formation. (F) Transfection of miR-509-5p inhibitor significantly promoted A549 cells colony formation. Data represent mean \pm SE $(\mathrm{n}=3)$. ${ }^{*}, P<0.05 ;{ }^{* *}, P<0.01 ;{ }^{* * *}, P<0.001$, compared with the negative control.

viability in NSCLC cells. The miR-509-5p mimics were transfected into H1299 cells with relative low miR-509-5p expression. Forced expression of miR-509-5p mimics in H1299 cells significantly increased miR-509-5p levels, whereas miR-509-5p inhibitor decreased miR-509-5p expression (Fig. 2A). By cell viability assay, we showed that miR-509-5p mimics obviously decreased cell viability in H1299 cells (Fig. 2C). Additionally, miR-509-5p mimics and inhibitor were transfected into A549 cells with relative high miR-509-5p expression. MiR-509-5p mimics and inhibitor significantly increased and abated miR-509-5p levels in 


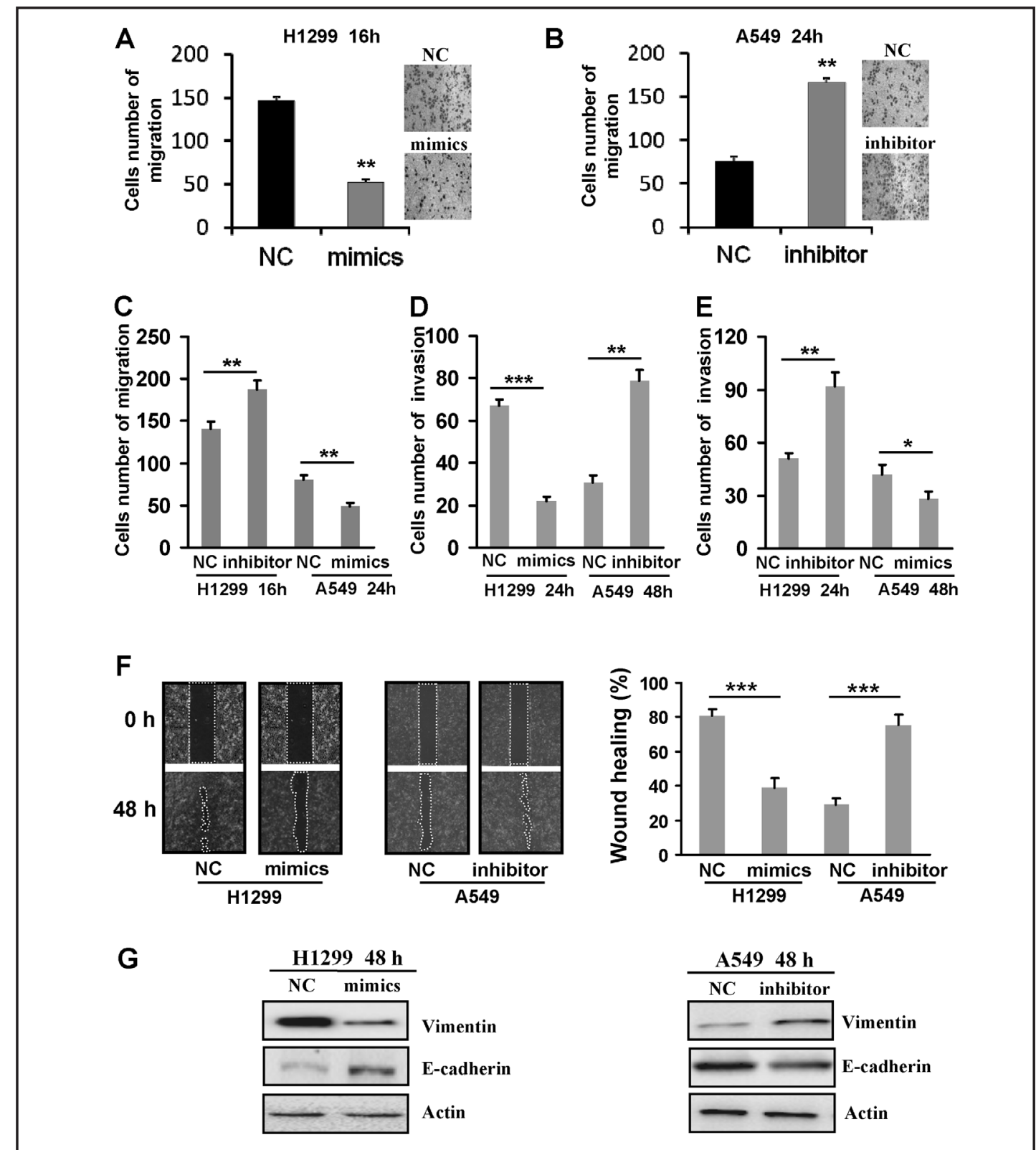

Fig. 3. miR-509-5p inhibits migration and invasion capability of NSCLC cells. (A) Migration assays were performed in H1299 cells transfected with miR-509-5p mimics and negative control (NC). (B) Migration assays were performed in A549 cells transfected with miR-509-5p inhibitor. (C) Migration assays were performed in H1299 cells transfected with miR-509-5p inhibitor, and A549 cells transfected with miR-509-5p mimics. (D) and (E) Invasion assays were performed in H1299 cells and A549 cells transfected with miR509-5p inhibitor and mimics. Data are mean \pm SE from three independent experiments. ${ }^{*}, P<0.05 ;{ }^{* *}, P<$ $0.01 ;{ }^{* * *}, P<0.001$, compared with NC. (F) The effects of miR-509-5p on NSCLC cell migration were determined by wound healing assay in $\mathrm{H} 1299$ and A549 cells (left lanel). Cells were seeded into 6-well plate and transfected with miR-509-5p mimics or inhibitor, and then subjected to wound healing assay. The level of cell migration into the wound scratch (dotted lines) was quantified as the percentage of wound sealing and compared against that of control cells at each time point (right lanel). ${ }^{* * *}, P<0.001$, compared with NC. (G) Western blot analysis of E-cadherin and vimentin in H1299 transfected with miR-509-5p mimics and A549 cells transfected with miR-509-5p inhibitor. Actin was used as a loading control.

A549 cells respectively(Fig. 2B). Moreover, A549 cells viability was obviously enhanced by miR-509-5p inhibitor, whereas reduced by miR-509-5p inhibitor (Fig. 2D). Furthermore, 
Fig. 4. miR-509-5p directly targets FOXM1. The mRNA expression $(A)$ and protein level (B) of FOXM1 were downregulated by miR-5095p in H1299 and A549 cells. H1299 and A549 cells were transfected with miR-509$5 p$ mimics or miR-509-5p inhibitor and the negative control (NC), respectively. RT-PCR was performed to detect the mRNA expression of miR-509-5p. Western blot was performed to detect the protein expression of FOXM1. Actin was used as a loading control. (C) The predicted miR-509-5p target site in the 3'UTR of FOXM1 mRNA and its mutated version. (D) and (E) Luciferase activity assays in 293T and A549 cells showed that miR509-5p inhibited the expression of FOXM1. Data were from three independent experiments. Data are mean \pm SE from three independent experiments. $* *, P<0.01$, compared with the control group.

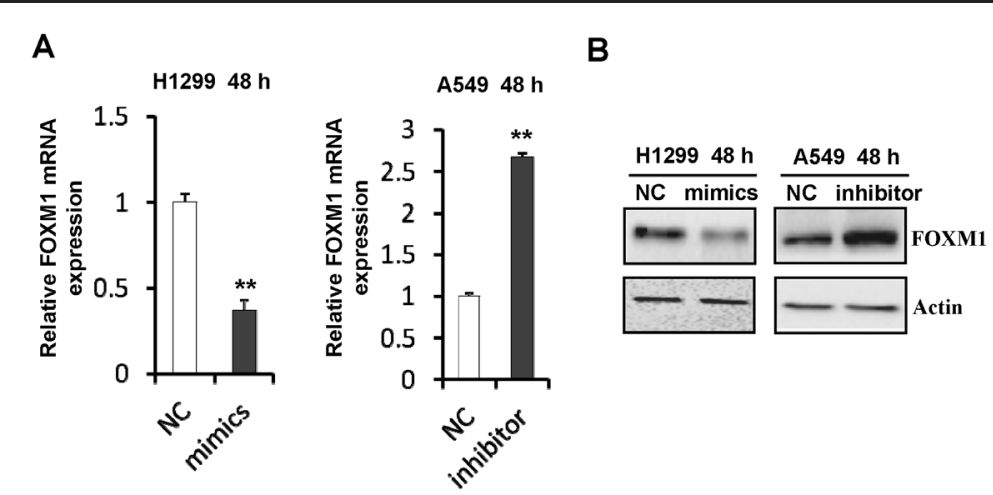

C

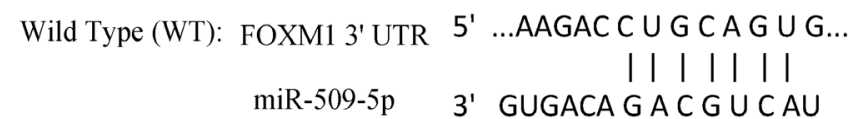

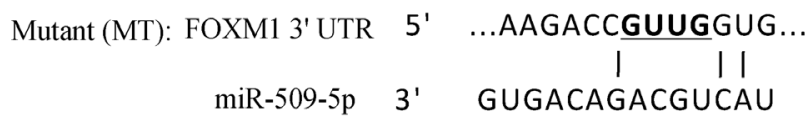

D
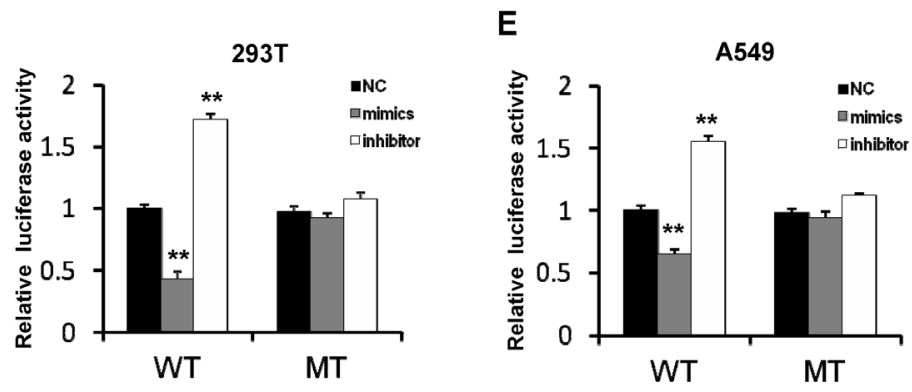

by cell colony formation assay, we found that miR-509-5p mimics apparently inhibited the clonogenic activity of H1299 cells (Fig. 2E). In contrast, the clonogenic activity of A549 cells was significantly enhanced by miR-509-5p inhibitors (Fig. 2F).

\section{miR-509-5p inhibits migration and invasion capability of NSCLC cells}

To explore the role of miR-509-5p in the migration and invasion of NSCLC cells, we further examined the effects of miR-509-5p on cell migration and invasion in H1299 and A549 cells by transiently transfection with miR-509-5p mimics or miR-509-5p inhibitors. Transwell assay showed that miR-509-5p mimics significantly reduced cell migration in H1299 and A549 cells; conversely, miR-509-5p inhibitor obviously enhanced H1299 and A549 cells migration and invasion (Fig. 3A-C). Moreover, by invasion assay, we showed the effects of A549 and H1299 cells invasion were observably suppressed by miR-509-5p mimics and increased by miR-509-5p inhibitor (Fig. 3D-E). In addition, wound healing assay indicated that miR-509-5p mimics transfected H1299 cells migrated into the wound area slower than control cells (Fig. 3F). In contrast, we found miR-509-5p inhibitor promoted A549 cells migration (Fig. 3F).

E-cadherin is negatively correlated with increased invasion and migration capability of cells [26], whereas vimentin induces cells migration and invasion [27]. To investigate the effects of miR-509-5p on E-cadherin and vimentin expression, we investigated the protein expression of the two molecules in H1299 cells transfected miR-509-5p mimics and A549 cells transfected miR-509-5p inhibitor respectively. By Western blot analyses, we found miR- 


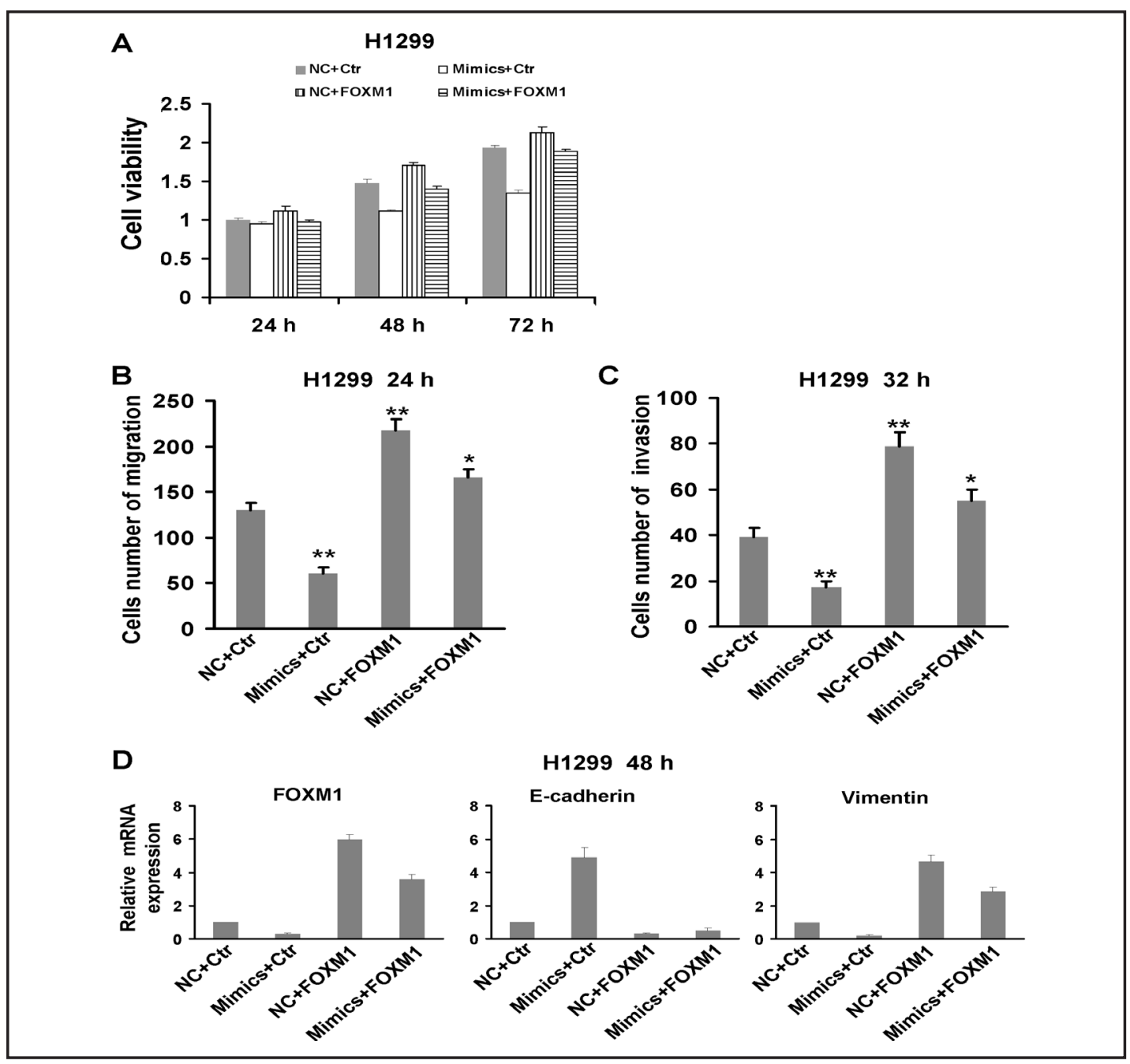

Fig. 5. Overexpression of FOXM1 reverses cell viability, migration, invasion and vimentin levels suppressed by miR-509-5p mimics in H1299 cells. H1299 cells were cotransfected with miR-509-5p mimics and FOXM1 vectors. (A) MTT assay was performed to examine cell viability after transfection at 24,48 and $72 \mathrm{~h}$, respectively. (B) and (C) Migration and invasion assays were performed in H1299 cells. Data are mean \pm SE from three independent experiments. ${ }^{*}, P<0.05$; ${ }^{*}, P<0.01$, compared with NC. (D) RT-PCR was performed to detect the expression of FOXM1, E-cadherin and vimentin.

509-5p mimics decreased vimentin levels and increased E-cadherin expression in H1299 cells (Fig. 3G left panel). In contrast, vimentin levels were induced and E-cadherin levels were reduced by miR-509-5p inhibitor in A549 cells respectively (Fig. 3G right panel).

\section{miR-509-5p directly targets FOXM1}

Using online miRNA target prediction databases (PicTar, miRDB and TargetScan), we predicted FOXM1 was a potential target of miR-509-5p. To validate targeting of FOXM1 by miR-509-5, the mRNA and protein levels of FOXM1 were detected in NSCLC cells transfected miR-509-5p mimics and inhbitor. MiR-509-5p mimics significantly attenuated the protein and mRNA levels of FOXM1 in H1299 cells, whereas miR-509-5p inhibitor observably enhanced FOXM1 protein and mRNA levels (Fig. 4A and B). To further confirm targeting of FOXM1 by miR-509-5p, luciferase activity assay was performed. The wild type (WT) or mutated (MT) FOXM1 3 'UTR luciferase reporter vectors were cotransfected with miR-509$5 \mathrm{p}$ mimics or inhibitor into $293 \mathrm{~T}$ and A549 cells. MiR-509-5p mimics significantly decreased luciferase activity in both 293T and A549 cells transfected WT reporter vectors (Fig. 4D and 
Fig. 6. miR-509-5p is negatively correlated with FOXM1 mRNA expression. (A) FOXM1 is highly expressed in human NSCLC tissues. Expression of FOXM1 was determined by RT-PCR in 20 paired human NSCLC samples and their corresponding adjacent normal samples, and $\beta$-actin as control. The experiments were repeated three times. (B) and (C) Significant negative correlation was found between miR509-5p and FOXM1 in human NSCLC and adjacent normal tissues. (D) Significant positive correlation was found between vimentin and FOXM1 in human NSCLC tissues. (E) Significant negative correlation was found between vimentin and miR-509-5p in human NSCLC tissues.
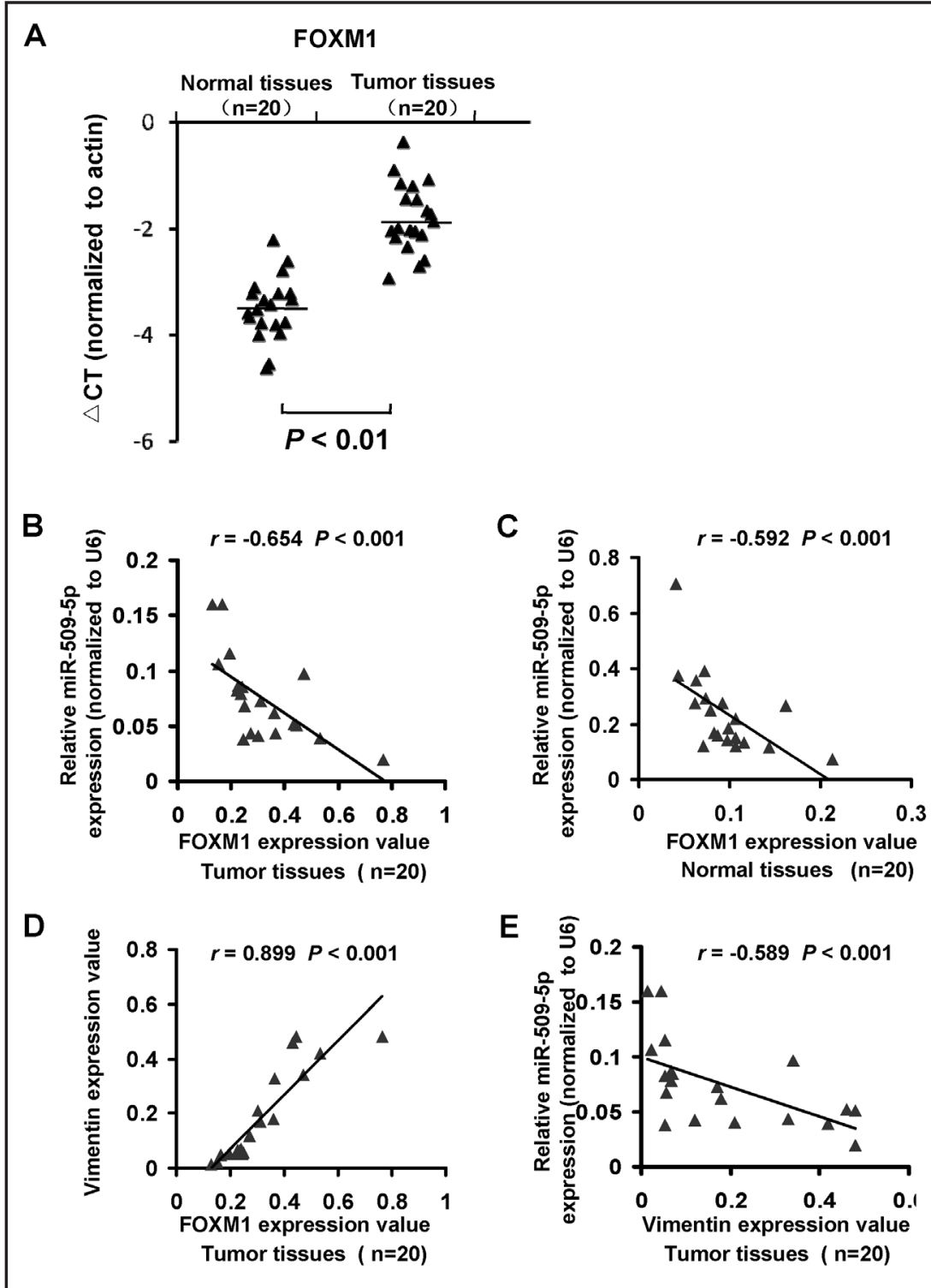

E). However no obvious change of luciferase activity was observed in both 293T and A549 cells transfected MT reporter vectors (Fig. 4D and E). Above results implied that miR-509-5p suppressed FOXM1 expression by binding 3 'UTR of FOXM1 mRNA.

Overexpression of FOXM1 reverses cell viability, migration, invasion and vimentin levels suppressed by miR-509-5p mimics in $H 1299$ cells

To envaluate if FOXM1 is responsible for the functional effects of miR-509-5p in NSCLC cells, we performed some rescue experiments. H1299 cells were co-transduced with miR509 mimics plus the eukaryotic expression vectors of FOXM1 open reading frame without its 3'UTR. And then, cell viability, migration and invasion assays were performed. H1299 cells co-transduced with miR-509-5p mimics plus FOXM1 vectors had higher cell viability than cells co-transduced with the control mimics and empty vectors (Fig. 5A). Additionally, we showed the decrease of cell migration and invasion by miR-509-5p mimics were reversed by FOXM1 in H1299 cells (Fig. 5B and C). Moreover, by RT-PCR, the downregulation of vimentin and upregulation of E-cadherin induced by miR-509-5p mimics were rescued by FOXM1 in the cell lines (Fig. 5D). 


\section{Cellular Physiology Cell Physiol Biochem 2016;38:1435-1446

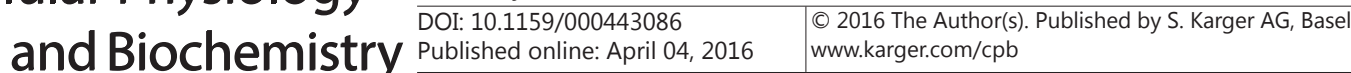 \\ Ma et al.: MiRNA-509-5p Inhibits NSCLC by Targeting FOXM1}

miR-509-5p is negatively correlated with FOXM1 mRNA expression

To further examine whether miR-509-5p expression was correlated with FOXM1, we analyzed FOXM1 mRNA levels in the 20 paired specimens by qRT-PCR. We showed that NSCLC tissue specimens exhibited higher FOXM1 levels compared with adjacent normal tissue specimens (Fig. 6A). Moreover, by correlation analysis between miR-509-5p and FOXM1, we found significantly negative correlations between miR-509-5p and FOXM1 mRNA expression in both NSCLC and adjacent normal tissue specimens (Fig. 6B, for tumor tissue: $r=-0.654$; Fig. 6C, for normal tissue: $r=-0.592$ ). In addition, to examine whether miR509-5p expression was correlated with vimentin, we analyzed vimentin mRNA levels in the 20 tumor specimens by qRT-PCR. By correlation analysis, a significantly positive correlation was found between FOXM1 and vimentin (Fig. 6D, $r=0.899$ ), and a significantly negative correlation was found between miR-509-5p and vimentin (Fig. 6E, $r=-0.589$ ).

\section{Discussion}

MiRNAs have been reported to function as cancer activators or suppressors in a variety of human tumors including NSCLC [28]. The upregulation or downregulation of miRNAs levels found in several human cancers suggests that miRNAs may induce or inhibit tumorigenesis. miRNAs have been implicated in various cellular processes including cell proliferation, survival, apoptosis, migration, invasion and differentiation [29, 30]. miR-509$5 \mathrm{p}$ has been implicated in cervical cancer and hepatocellular carcinoma, playing important roles in inhibition of tumorigenesis by abrogating cancer cell proliferation and invasion [18]. Moreover, miR-509-5p induces p53 protein levels that subsequently arrest cell cycle and increase cell apoptosis by directly targeting HDM2 in cervical cancer and hepatocellular carcinoma cells [18]. Additionally, it has reported that miR-509-5p expression in renal cell carcinoma specimens is obviously decreased compared with adjacent normal tissue [17]. Furthermore, the proliferation and migration of renal cell carcinoma cells is suppressed by miR-509-5p, whereas cell apoptosis is induced by miR-509-5p [17]. Consistent with these findings, in the present study, we found miR-509-5p levels were down-regulated in NSCLC tissues compared with the adjacent normal tissues. This was, to our knowledge, the first study showing miR-509-5p was low expressed in NSCLC. However, this conclusion needed to be further verified by expanding the numbers of sample. Additionally, we also found that miR-509-5p decreased cell viability and colony formation capability, and inhibited migration and invasion capability of NSCLC cells.

Generally, miRNAs display functions via binding to the mRNA3'-UTR of the target gene to block its expression [31]. To identify the target genes of miR-509-5p, more than 90 candidate target genes were predicted by online bioinformatics analysis. Combining the functions of prediction genes and the tumor suppressor actions of the miRNA described above, of these candidates, we focused on five genes (STAT5A, BCL9, FGF2, MAPRE2 and FOXM1). Previous studies report these five genes function as oncogene. However, only FOXM1 has been shown to involved in cell migration, invasion and tumorigenesis of NSCLC. Therefore, we selected FOXM1 as a target gene of miR-509-5p for further study. Next, by dual-luciferase reporter assay, we validated FOXM1 was a target of the miRNA. Lastly, we further showed miR-509-5p was negatively correlated with FOXM1 mRNA expression in both NSCLC and normal tissues. Recent studies indicate that miRNAs are involved in regulation of FOXM1 expression [32]. miR-134, for example, has been implicated to regulate NSCLC cells migration and invasion by targeting FOXM1 [33].

FOXM1, a member of the forkhead box family of transcriptional factors, has been reported to be overexpressed in a variety of cancers including breast, cervical, colorectal and ovarian cancer, hepatocellular carcinoma and NSCLC [34, 35]. Moreover, overexpression of FOXM1 has been linked in poor prognosis in many solid tumor types [7, 36]. Previous studies show FOXM1 enhances cell cycle progression by promoting G1 to $\mathrm{S}$ and G2 to M phase transitions [37]. In addition, downregulation of FOXM1 levels has been reported to decrease migration and invasion of cancer cells [38]. Moreover, it has been demonstrated 


\section{Cellular Physiology Cell Physiol Biochem 2016;38:1435-1446

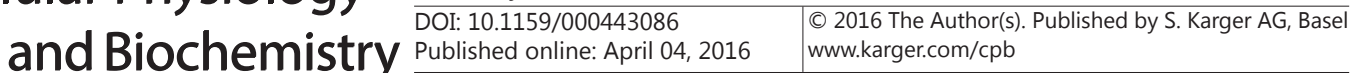 \\ Ma et al.: MiRNA-509-5p Inhibits NSCLC by Targeting FOXM1}

that FOXM1 enhances EMT of cancer cells by increasing the levels of vimentin, N-cadherin and beta-catenin, decreasing the levels of E-cadherin [39]. Here, overexpression of miR-509$5 p$ mimics induced E-cadherin expression and reduced vimentin levels, whereas miR-509$5 p$ inhibitor attenuated E-cadherin expression and increased vimentin levels. This raised a possibility that miR-509-5p regulated the expression of E-cadherin and vimentin by targeting FOXM1.

Altogether, the present data provided further evidence that miR-509-5p might be considered as a novel and potential target for the diagnosis, prognosis and treatment of NSCLC.

\section{Acknowledgements}

This study was supported by grants from the First Affiliated Hospital of Zhengzhou University Science Fund for Young Scholars (to N. Ma).

\section{Disclosure Statement}

The authors declare that they have no conflict of interest.

\section{References}

1 Torre LA, Bray F, Siegel RL, Ferlay J, Lortet-Tieulent J, Jemal A: Global cancer statistics, 2012. CA Cancer J Clin 2015;65:87-108.

2 Molina JR, Yang P, Cassivi SD, Schild SE, Adjei AA: Non-small cell lung cancer: epidemiology, risk factors, treatment, and survivorship. Mayo Clin Proc 2008;83:584-594.

3 Paez JG, Janne PA, Lee JC, Tracy S, Greulich H, Gabriel S, Herman P, Kaye FJ, Lindeman N, Boggon TJ, Naoki K, Sasaki H, Fujii Y, Eck MJ, Sellers WR, Johnson BE, Meyerson M: EGFR mutations in lung cancer: correlation with clinical response to gefitinib therapy. Science 2004;304:1497-1500.

4 Eccles SA, Welch DR: Metastasis: recent discoveries and novel treatment strategies. Lancet 2007;369:17421757.

5 He L, Hannon GJ: MicroRNAs: small RNAs with a big role in gene regulation. Nat Rev Genet 2004;5:522531.

6 Calin GA, Croce CM: MicroRNA signatures in human cancers. Nat Rev Cancer 2006;6:857-866.

7 Miyashita A, Fukushima S, Nakahara S, Yamashita J, Tokuzumi A, Aoi J, Ichihara A, Kanemaru H, Jinnin M, Ihn H: Investigation of FOXM1 as a Potential New Target for Melanoma. PLoS One 2015;10:e144241.

8 Kasinski AL, Slack FJ: Epigenetics and genetics. MicroRNAs en route to the clinic: progress in validating and targeting microRNAs for cancer therapy. Nat Rev Cancer 2011;11:849-864.

9 Li J, Yu T, Cao J, Liu L, Liu Y, Kong HW, Zhu MX, Lin HC, Chu DD, Yao M, Yan MX: MicroRNA-148a Suppresses Invasion and Metastasis of Human Non-Small Cell Lung Cancer. Cell Physiol Biochem 2015;37:1847-1856.

10 He Z, Xia Y, Pan C, Ma T, Liu B, Wang J, Chen L, Chen Y: Up-Regulation of MiR-452 Inhibits Metastasis of Non-Small Cell Lung Cancer by Regulating BMI1. Cell Physiol Biochem 2015;37:387-398.

11 Deng W, Yan M, Yu T, Ge H, Lin H, Li J, Liu Y, Geng Q, Zhu M, Liu L, He X, Yao M: Quantitative proteomic analysis of the metastasis-inhibitory mechanism of miR-193a-3p in non-small cell lung cancer. Cell Physiol Biochem 2015;35:1677-1688.

12 Zhang L, Qian J, Qiang Y, Huang H, Wang C, Li D, Xu B: Down-regulation of miR-4500 promoted non-small cell lung cancer growth. Cell Physiol Biochem 2014;34:1166-1174.

13 Hu X, Zhang F, Liu XR, Wu YT, Ni YM: Efficacy and potential microRNA mechanism for computed tomography-guided percutaneous radiofrequency ablation of primary lung cancer and lung metastasis from liver cancer. Cell Physiol Biochem 2014;33:1261-1271.

14 Wu N, Zhang C, Bai C, Han YP, Li Q: MiR-4782-3p inhibited non-small cell lung cancer growth via USP14. Cell Physiol Biochem 2014;33:457-467.

15 Dong W, Yongjun L, Nan D, Junyun W, Qiong Y, Yaran Y, Yanming L, Xiangdong F, Hua Z: Molecular networks and mechanisms of epithelial-mesenchymal transition regulated by miRNAs in the malignant melanoma cell line. Yi Chuan 2015;37:673-682. 


\section{Cellular Physiology Cell Physiol Biochem 2016;38:1435-1446 \begin{tabular}{ll|l} 
and Biochemistry $\begin{array}{l}\text { DOI.1159/000443086 } \\
\text { Published online: April 04, } 2016\end{array}$ & $\begin{array}{l}\text { C } 2016 \text { The Author(s). Published by S. Karger AG, Basel } \\
\text { www.karger.com/cpb }\end{array}$
\end{tabular} \\ Ma et al.: MiRNA-509-5p Inhibits NSCLC by Targeting FOXM1}

16 Xing F, Sharma S, Liu Y, Mo YY, Wu K, Zhang YY, Pochampally R, Martinez LA, Lo HW, Watabe K: miR509 suppresses brain metastasis of breast cancer cells by modulating RhoC and TNF-alpha. Oncogene 2015;34:4890-4900.

17 Zhang WB, Pan ZQ, Yang QS, Zheng XM: Tumor suppressive miR-509-5p contributes to cell migration, proliferation and antiapoptosis in renal cell carcinoma. Ir J Med Sci 2013;182:621-627.

18 Ren ZJ, Nong XY, Lv YR, Sun HH, An PP, Wang F, Li X, Liu M, Tang H: Mir-509-5p joins the Mdm2/p53 feedback loop and regulates cancer cell growth. Cell Death Dis 2014;5:e1387.

19 Gao J, Zou Z, Gao J, Zhang H, Lin Z, Zhang Y, Luo X, Liu C, Xie J, Cai C: Increased expression of HMGB3: a novel independent prognostic marker of worse outcome in patients with esophageal squamous cell carcinoma. Int J Clin Exp Pathol 2015;8:345-352.

20 Nie P, Hu W, Zhang T, Yang Y, Hou B, Zou Z: Synergistic Induction of Erlotinib-Mediated Apoptosis by Resveratrol in Human Non-Small-Cell Lung Cancer Cells by Down-Regulating Survivin and Up-Regulating PUMA. Cell Physiol Biochem 2015;35:2255-2271.

21 Zou Z, Yuan Z, Zhang Q, Long Z, Chen J, Tang Z, Zhu Y, Chen S, Xu J, Yan M, Wang J, Liu Q: Aurora kinase A inhibition-induced autophagy triggers drug resistance in breast cancer cells. Autophagy 2012;8:1798-1810.

22 Jiang S, Zou Z, Nie P, Wen R, Xiao Y, Tang J: Synergistic Effects between mTOR Complex 1/2 and Glycolysis Inhibitors in Non-Small-Cell Lung Carcinoma Cells. PLoS One 2015;10:e132880.

23 Mi YJ, Geng GJ, Zou ZZ, Gao J, Luo XY, Liu Y, Li N, Li CL, Chen YQ Yu XY, Jiang J: Dihydroartemisinin inhibits glucose uptake and cooperates with glycolysis inhibitor to induce apoptosis in non-small cell lung carcinoma cells. PLoS One 2015;10:e120426.

24 Lou YF, Zou ZZ, Chen PJ, Huang GB, Li B, Zheng DQ Yu XR, Luo XY: Combination of gefitinib and DNA methylation inhibitor decitabine exerts synergistic anti-cancer activity in colon cancer cells. PLoS One 2014;9:e97719.

25 Zou ZZ, Nie PP, Li YW, Hou BX, Rui-Li, Shi XP, Ma ZK, Han BW, Luo XY: Synergistic induction of apoptosis by salinomycin and gefitinib through lysosomal and mitochondrial dependent pathway overcomes gefitinib resistance in colorectal cancer. Oncotarget DOI: 10.18632/oncotarget.5628.

26 Durer U, Hartig R, Bang S, Thim L, Hoffmann W: TFF3 and EGF induce different migration patterns of intestinal epithelial cells in vitro and trigger increased internalization of E-cadherin. Cell Physiol Biochem 2007;20:329-346.

27 Hanahan D, Weinberg RA: Hallmarks of cancer: the next generation. Cell 2011;144:646-674.

28 Rolfo C, Fanale D, Hong DS, Tsimberidou AM, Piha-Paul SA, Pauwels P, Van Meerbeeck JP, Caruso S, Bazan V, Cicero G, Russo A, Giovannetti E: Impact of microRNAs in resistance to chemotherapy and novel targeted agents in non-small cell lung cancer. Curr Pharm Biotechnol 2014;15:475-485.

29 Chang L, Shrestha S, LaChaud G, Scott MA, James AW: Review of microRNA in osteosarcoma and chondrosarcoma. Med Oncol 2015;32:613.

30 Bracken CP, Khew-Goodall Y, Goodall GJ: Network-Based Approaches to Understand the Roles of miR-200 and other microRNAs in Cancer. Cancer Res 2015;75:2594-2599.

31 Bartel DP: MicroRNAs: genomics, biogenesis, mechanism, and function. Cell 2004;116:281-297.

32 Shi M, Cui J, Xie K: Signaling of miRNAs-FOXM1 in cancer and potential targeted therapy. Curr Drug Targets 2013;14:1192-1202.

33 Li J, Wang Y, Luo J, Fu Z, Ying J, Yu Y, Yu W: miR-134 inhibits epithelial to mesenchymal transition by targeting FOXM1 in non-small cell lung cancer cells. FEBS Lett 2012;586:3761-3765.

34 Bella L, Zona S, Nestal DMG, Lam EW: FOXM1: A key oncofoetal transcription factor in health and disease. Semin Cancer Biol 2014;29:32-39.

35 Fan Q, Cai Q, Xu Y: FOXM1 is a downstream target of LPA and YAP oncogenic signaling pathways in high grade serous ovarian cancer. Oncotarget 2015;6:27688-27699.

36 Xu K, Liu X, Mao X, Xue L, Wang R, Chen L, Chu X: MicroRNA-149 suppresses colorectal cancer cell migration and invasion by directly targeting forkhead box transcription factor FOXM1. Cell Physiol Biochem 2015;35:499-515.

37 Anders L, Ke N, Hydbring P, Choi YJ, Widlund HR, Chick JM, Zhai H, Vidal M, Gygi SP, Braun P, Sicinski P: A systematic screen for CDK4/6 substrates links FOXM1 phosphorylation to senescence suppression in cancer cells. Cancer Cell 2011;20:620-634.

38 Bowman A, Nusse R: Location, location, location: FoxM1 mediates beta-catenin nuclear translocation and promotes glioma tumorigenesis. Cancer Cell 2011;20:415-416.

39 Valastyan S, Weinberg RA: Tumor metastasis: molecular insights and evolving paradigms. Cell 2011;147:275-292. 